\title{
La terapia puente durante la interrupción de la warfarina se asoció a un mayor riesgo de sangrado
}

\author{
-Bridge therapy during warfarin discontinuation was associated with an increased risk of bleeding
}

Clarke NP y col. JAMA Intern Med. 2015;175(7):1163-1168.

\begin{abstract}
Objetivos
Describir la incidencia de hemorragias clínicamente relevantes y enfermedad tromboembólica venosa recurrente - definida como trombosis venosa profunda (TVP) de la extremidad superior o inferior y/o embolia pulmonar- en pacientes que interrumpen la terapia con warfarina para realizar procedimientos invasivos, comparando dicha incidencia entre quienes recibieron terapia "puente" con heparina y quienes no, durante la interrupción.
\end{abstract}

\section{Diseño, lugar y pacientes}

Cohorte retrospectiva llevado adelante en "Kaiser permanente, Colorado" (sistema integrado de prestación de servicios de salud), Estados Unidos. Incluyó adultos sometidos a un procedimiento invasivo diagnóstico o quirúrgico entre 2006 y 2012, que habían interrumpido la terapia con warfarina que estaban recibiendo para la prevención secundaria de ETV -sin fibrilación auricular ni válvula cardíaca mecánica-, que al momento de la interrupción tenían una razón internacional normalizada mayor o igual a 1,5 , que reanudaron el tratamiento con warfarina durante los 30 días posteriores al procedimiento, y que no tuvieron otra interrupción del tratamiento relacionada con el procedimiento durante los 90 días posteriores al procedimiento índice.

\section{Evaluación de variables}

La variable de exposición fue el uso de la terapia puente -uso de un anticoagulante de acción corta, típicamente heparina de bajo peso molecular, durante el periprocedimiento-, versus ninguna terapia durante la interrupción de la warfarina. La de resultado fue el sangrado clínicamente relevante, ETV recurrente y mortali- dad global, verificados mediante revisión manual de los registros médicos.

\section{Resultados principales}

Entre los 1178 pacientes, la edad media fue 66,1 años, $45,8 \%$ fueron hombres y la indicación más común de warfarina fue TVP $(56,3 \%)$. La mayoría tenía bajo riesgo de recurrencia de ETV al momento de la interrupción (79 \%, 1431 procedimientos) de acuerdo con las pautas de consenso del American College of Chest Physicians. 15 pacientes $(2,7 \%)$ del grupo terapia puente y dos $(0,2 \%)$ del grupo sin ella desarrollaron hemorragias clínicamente relevantes durante los 30 días posteriores al procedimiento (RR 17,2 , IC $95 \% 3,9$ a 75,1). No hubo muertes ni diferencias significativas en la tasa de ETV recurrente entre los grupos ( 0 versus 3 respectivamente; $\mathrm{p} 0,56$ ).

\section{Conclusiones}

La terapia puente se asoció con un mayor riesgo de sangrado durante la interrupción de warfarina para procedimientos invasivos en pacientes que reciben tratamiento por ETV previa y probablemente su indicación sea innecesaria. Se necesitan más investigaciones que permitan identificar las características del paciente y del procedimiento asociadas con un alto riesgo de recidiva perioperatoria de ETV durante la interrupción del tratamiento con warfarina.

Conflicto de interés: EI Dr. Douketis fue consultor para uso clínico de fármacos antiplaquetarios (ticagrelor) y fármacos anticoagulantes (apixaban, dabigatran y semuloparina). Fuente de financiamiento: Este estudio fue realizado y financiado por Kaiser Permanente Colorado.

\section{Comentario}

Este estudio documentó que el uso de terapia puente con anticoagulantes de acción corta para procedimientos invasivos en pacientes que reciben tratamiento por ETV previa con warfarina ${ }^{1}$ se asoció con un mayor riesgo de sangrado sin cambios en la incidencia de recurrencia, lo que hace pensar que su indicación sería innecesaria. Si bien se trata de información que proviene de una gran base de datos secundaria de alta calidad, estas conclusiones deben ser interpretadas con cautela ${ }^{2}$ ya que quienes reciben anticoagulación por ETV previa tienen riesgos variables de recurrencia al suspender el tratamiento, siendo el determinante más importante el tiempo transcurrido desde el evento primario ${ }^{3}$, aunque el cáncer activo o los estados de hipercoagulabilidad son otras variables a considerar. La suspensión durante las primeras cuatro semanas implica un riesgo diario de ETV recurrente de 0,3 a $1,3 \%$, disminuyendo a 0,03 a $0,2 \%$ por día durante las semanas cuatro a 12. Después de tres meses la mayoría podría suspender la anticoagulación con buena seguridad. Vale desta- car que casi el $80 \%$ de los pacientes incluidos en este estudio tenían bajo riesgo de recurrencia (1431/1812) y sólo un tercio había recibido terapia puente dado que su indicación no está sistematizada. Por un lado, como sugiere la $\mathrm{ACC}^{4}$ es importante estratificar a los pacientes que están recibiendo anticoagulación por ETV de acuerdo a su riesgo de recurrencia para determinar la necesidad de terapia puente; y por otro, dado que esta población fue mayormente de bajo riesgo, es difícil extrapolar dicha información a una conducta global.

\section{Conclusiones de los comentadores}

La terapia puente puede ser evitada en pacientes de bajo riesgo de ETV recurrente. Sin embargo, resulta trascendental evaluar en cada caso particular el riesgo hemorrágico del procedimiento versus el riesgo de recurrencia, para así evitar el sobreuso ${ }^{5}$ o la subutilización de anticoagulantes parenterales en este escenario clínico.

María Florencia Grande Ratti [ Servicio de Medicina Familiar y Comunitaria del Hospital Italiano de Buenos Aires, Área de Investigación en Medicina Interna HIBA. maria.grande@ hospitalitaliano.org.ar ] y Juan Farina [ Sanatorio de la Trinidad Mitre. juan.farina@gmail.com ]

Grande Ratti M y Farina J. La terapia puente durante la interrupción de la warfarina se asoció con un mayor riesgo de sangrado. Evid Act Pract Ambul. 2017(4): 91. Comentado de: Clarke NP y col. Bleeding, Recurrent Venous Thromboembolism, and Mortality Risks During Warfarin Interruption for Invasive Procedures. JAMA Intern Med. 2015; 175(7):1163-1168.

Referencias bibliográficas

1 Jaffer A al. When patients on warfarin need surgery. Cleve Clin J Med. 2003 Nov; 70(11):973-84

2. Grande Ratti M. Estudios de cohorte. Revista Hospital Italiano Buenos Aires. 2016(36(1)):17-8.

3. Siegal $\mathrm{D}$ y col. Periprocedural heparin bridging in patients receiving vitamin $\mathrm{K}$ antagonists: systematic review and meta-analysis of bleeding and thromboembolic rates. Circulation. 2012 Sep 25; 126(13):1630-9.

4. Douketis J al. Perioperative management of antithrombotic therapy: Antithrombotic Therapy and Prevention of Thrombosis, 9th ed: American College of Chest Physicia... PubMed - NCBI [Internet]. [cited 2017 May 16]. Available from: https://www.ncbi.nlm.nih.gov/pubmed/22315266

5. Brotman D. Overuse of Bridging Anticoagulation for Patients With Venous Thromboembolism. JAMA Intern Med. 2015; 175(7):1169. 